\title{
Low Compliance with Alcohol Gel Compared with Chlorhexidine for Hand Hygiene in ICU Patients: Results of an Alcohol Gel Implementation Program
}

\author{
Luis Fernando Aranha Camargo, Alexandre Rodrigues Marra, Cláudia Vallone Silva, Cláudia Regina Laselva, Denis Faria \\ Moura Junior, Ruy Guilherme G. Cal, Maria Aparecida Yamashita and Elias Knobel \\ Hospital Israelita Albert Einstein, Albert Einstein Avenue 627, Morumbi; São Paulo, SP, Brazil
}

\begin{abstract}
Although the introduction of alcohol based products have increased compliance with hand hygiene in intensive care units (ICU), no comparative studies with other products in the same unit and in the same period have been conducted. We performed a two-month-observational prospective study comparing three units in an adult ICU, according to hand hygiene practices (chlorhexidine alone-unit $\mathrm{A}$, both chlorhexidine and alcohol gel-unit $\mathrm{B}$, and alcohol gel alone-unit $\mathrm{C}$, respectively). Opportunities for hand hygiene were considered according to an institutional guideline. Patients were randomly allocated in the 3 units and data on hand hygiene compliance was collected without the knowledge of the health care staff. TISS score (used for measuring patient complexity) was similar between the three different units. Overall compliance with hand hygiene was $46.7 \%$ (659/1410). Compliance was significantly higher after patient care in unit A when compared to units B and C. On the other hand, compliance was significantly higher only between units A $(32.1 \%)$ and C $(23.1 \%)$ before patient care $(p=0.02)$. Higher compliance rates were observed for general opportunities for hand hygiene (patient bathing, vital sign controls, etc), while very low compliance rates were observed for opportunities related to skin and gastroenteral care. One of the reasons for not using alcohol gel according to health care workers was the necessity for water contact $(35.3 \%, 12 / 20)$. Although the use of alcohol based products is now the standard practice for hand hygiene the abrupt abolition of hand hygiene with traditional products may not be recommended for specific services.
\end{abstract}

Key-Words: Alcohol gel and chlorhexidine, Hand washing, ICU.

Hand hygiene is the cornerstone measure for nosocomial infection prevention in Intensive Care Units (ICU's) [1]. The use of soap or antiseptics are both effective for removal of transient skin flora. On the other hand, for effective removal of microorganisms in deeper sites of human normal skin an antiseptic is required [2].

Health care workers usually comply poorly with hand hygiene practices. Main reasons for such low compliance rates are side effects related to chemical products, lack of time due to excess workload and unawareness of the benefits of such practices. Rates bellow $50 \%$ have been reported worldwide and the lowest rates are usually reported in ICU's [3-5].

Multivariate analysis in ICU's have shown that excessive workload, i.e, excess of opportunities for hand hygiene due to complexity of patient care is independently associated with low compliance in such units [6]. Hence, along with educational policies, strategies to reduce the time spent with hand disinfection have been required to enhance compliance rates.

Alcohol based products that dispense the routine use of water have been shown to reduce the time spent with hand hygiene, while achieving even higher rates of hand decontamination when compared to commonly used products [7]. Early studies in Europe and the United States have shown a beneficial effect of employing such products [3,8], with

Received on 6 March 2009; revised 10 September 2009.

Address for correspondence: Dr.Luis Fernando Aranha Camargo. Rua Barão de Santa Eulália 170, ap.21. Zip code: 05685-090. São Paulo, SP, Brazil. Phone: 5511 37471500. Fax: 5511 37422834.E-mail: luisfacamargo@uol.com.br.

The Brazilian Journal of Infectious Diseases 2009;13(5):330-334. (C) 2009 by The Brazilian Journal of Infectious Diseases and Contexto Publishing. All rights reserved. increasing rates being observed with continued use along with educational and motivational strategies. Such studies, however, have used baseline compliance rates before introduction of alcoholic products as controls and no study has compared compliance rates between alcohol and conventional antiseptics in a contemporary way.

We have conducted a contemporary comparative study in different wards of the same general ICU, comparing health staff compliance to hand hygiene with alcohol gel, chlorhexidine or both.

The purpose of this study was to evaluate and to compare the compliance of hand hygiene in adult ICU for two different products: $2 \%$ chlorhexidine and alcohol gel.

\section{Material and Methods}

This study was conducted in the Intensive Care Unit of a tertiary care private Hospital in São Paulo, Brazil. The ICU is a 30-bed-medical-surgical unit. The beds are distributed in three 4-bed and 3 six-bed wards. The study was approved by the Hospital Ethics Committee. This study was conducted between August 2002 and February 2003. The 4-bed units, selected for the study, are composed of 4 individual boxes with dedicated material for patient care, such as stethoscopes and thermometers. There are 6 sinks ( 1 sink of each per bed and 2 outside the boxes), one nurse, one physician and one respiratory therapist and four technicians for patients care.

Antiseptic Use

Two percent chlorhexidine solution was being used for many years for hand hygiene at the time the study was conducted. The study comprised a two-month initial phase, where a general educational and motivational program was conducted, with practical demonstration of hand hygiene 
techniques, theoretical issues concerning benefits of hand hygiene and disclosure of compliance rates of hand hygiene in the ICU in past years. Special emphasis on theoretical and practical issues related to alcohol products (70\% ethyl alcohol) was demonstrated and introduction of such products for routine use was anticipated. Staff was particularly oriented on the dispensing of hand hygiene with alcohol products.

Following this one-month period, alcohol gel products were distributed in the different ICU wards and during a twomonth period, health care workers were encouraged to use the product. Study participants stayed at bedside to discuss staff questions regarding alcohol use and to emphasize that hand hygiene was not routinely necessary unless hand soiling was observed.

After this three-month period, the units were divided according to the antiseptic product employed: one unit used only $2 \%$ chlorhexidine (one dispenser per bed - UNIT A, representing our baseline hand hygiene compliance), one unit used both chlorhexidine and alcohol gel (1 dispenser of each per bed - UNIT B) and one unit used only alcohol gel at bedside (1 dispenser per bed - UNIT C), with a single chlorhexidine dispenser outside the individual boxes.

During the study period, patients were allocated according to bed availability in the units by the administrative staff, without the interference of nurses or physicians. The administrative staff was oriented to allocate patients irrespective of admission diagnosis and based on bed availability.

\section{Data Collection}

During a two-month-period, selected ICU staff members (not participating in the study) recorded data on hand hygiene compliance in the three assigned units. Data collection occurred during the whole working period and during the seven days of the week. Health staff was unaware of data recording.

Opportunities for hand hygiene were considered according to an institutional guideline. This guideline contains major recommendations in accordance with the Centers for Disease Control and Prevention guidelines for standard and contact precautions. Categorization of hand hygiene is shown in Table 1.

Data collection included patients under contact precautions due to multidrug-resistant organisms, intensity of nurse assistance (TISS score- Therapeutic Intervention Scoring System) and classification of hand hygiene opportunity according to category of patient care.

After the study termination, a questionnaire was elaborated to evaluate reasons for compliance/non-compliance to hand hygiene with alcohol products.

\section{Statistical Analysis}

Fisher exact test and Chi-square test were used to compare compliance hand hygiene rates between the three units, with a significance level of $<5 \%$. All statistical analyses were done using the Statistical Package for the Social Sciences software (SPSS, Chicago, IL, USA).

\section{Results}

During the 2-month data-collection phase of the study, 1410 opportunities for hand hygiene were evaluated, with 498 opportunities in unit A (only chlorhexidine), 454 opportunities in unit B (chlorhexidine and alcohol) and 458 opportunities in unit C (only alcohol).

Overall hand hygiene compliance was $46.7 \%$ (Table 2). General compliance was higher in unit A (56.4\%) and a significant difference was found between units A (56.4\%) and C (36.5\%) and between units B (46.5\%) and C (36.5\%) (for all comparisons, $\mathrm{p}<0.001)$. Hand hygiene was higher after (64.8\%) than before patient care (28.6\%), $\mathrm{p}<0.001$ in all units. Compliance was significantly higher after patient care in Unit A when compared to Units B and C. On the other hand, compliance was significantly higher only between units $\mathrm{A}$ (32.1\%) and C (23.1\%) before patient care, $\mathrm{p}=0.02$.

The total number of patients requiring contact isolation for multidrug- resistant pathogens was significantly higher in unit B (32\%) when compared to either units A (11.2\%) and C $(8.3 \%)(p=0.001)$. No statistically significant difference was observed between units A and C ( $>>0.05)$. TISS score was similar between units A, B and C, with no statistically significant difference (24.4, 27.9 and 23.1, respectively).

Higher compliance rates were observed for general opportunities for hand hygiene (patient bathing, vital sign controls etc), while very low compliance rates were observed for opportunities related to skin and gastroenteral care (Figure 1).

After the study completion, we asked 20 health-staff members about the reasons for refusal of hand-antisepsis with alcoholic gel (Table 3). One of the reasons for not using alcohol gel according to health care workers was the need for water contact $(35.3 \%, 12 / 20)$.

\section{Discussion}

The benefits of hand hygiene for patient care purposes are well known since Semmelweiss. However, several studies have consistently disclosed very low compliance rates of hand hygiene for procedures known or assumed to be related to prevention of nosocomial infections and transmission of infecting organisms between patients [911]. Compliance with hand hygiene in intensive care units (ICU) is the lowest among the different hospital units [6] and lower rates are observed before patient care [8]. Several reasons may be reported by health care workers to refuse hand hygiene, but only recently statistical and epidemiological tools were employed to analyze risk factors for non-compliance. Pittet et. al. have shown that compliance is inversely proportional to the number of opportunities for hand hygiene [6]. That means that more severe patients results in more busy health care workers and lower compliance, exactly what happens in ICU's. In fact, 
Table 1. Classification of hand antisepsis opportunities.

1 - Respiratory care: aspiration of tracheal secretions, respiratory therapist care, oral cavity hygiene, change of filters HME and respiratory circuits

2 - Catheter care: catheter insertion, IV line changes, drug administration, insertion site care, blood drawing

3 - Uro-genital care: urinary catheter insertion or removal, urine manipulation, urogenital antisepsis

4 - General care: bathing, scar care

5 - Nasogastric tube care: insertion or removal of nasogastric tube, measure of enteric drainage

6 - Others: physical examination, transportation of patients, etc.

Table 2. Hand antisepsis compliance rates according to study unit, considering general compliance as well as before and after patient care.

\begin{tabular}{lcccc}
\hline & UnitA & Unit B & Unit C & General \\
& N(\% compliance) & N (\% compliance) & N (\% compliance) & \\
\hline Before patient care & $80 / 249(32.1)^{*}$ & $69 / 227(30.4)^{\#}$ & $53 / 229(23.1)^{\#}$ & $202 / 705(28.6)$ \\
After patients care & $201 / 249(80.0)^{f}$ & $142 / 227(62.5)^{f}$ & $114 / 229(49.8)^{f}$ & $457 / 705(64.8)$ \\
Total compliance & $\mathbf{2 8 1 / 4 9 8 ~ ( 5 6 . 4 ) *}$ & $\mathbf{2 1 1 / 4 5 4}(\mathbf{4 6 . 5})^{*}$ & $\mathbf{1 6 7 / 4 5 8 ( 3 6 . 5 ) *}$ & $\mathbf{6 5 9 / 1 4 1 0 ( 4 6 . 7 )}$ \\
\hline
\end{tabular}

${ }^{*} \mathrm{p}<0.01 \mathrm{AxB}, \mathrm{AxC}$ and BxC; \# $\mathrm{p}=0.02 \mathrm{AxC}\left(\mathrm{p}>0.05\right.$ for AxB and BxC); ${ }^{f} \mathrm{p}<0.01 \mathrm{AxB}, \mathrm{AxC}$ and BxC.

Table 3. Reasons for not using alcohol gel according to health care workers.

\begin{tabular}{lcccc}
\hline & Hand drying & Need for water contact & Thick product & Total \\
\hline Physicians & $3(37.5 \%)$ & $3(37.5 \%)$ & $2(25 \%)$ & $8(100 \%)$ \\
Nurses & $1(16.7 \%)$ & $2(33.3 \%)$ & $3(50 \%)$ & $6(100 \%)$ \\
Technicians & $5(25 \%)$ & $7(35.7 \%)$ & $8(39 \%)$ & $20(100 \%)$ \\
Total & $9(26.4)$ & $12(35.3)$ & $13(38.2 \%)$ & $34(100 \%)$ \\
\hline
\end{tabular}

Figure 1. Hand antisepsis compliance according to category of patient care.

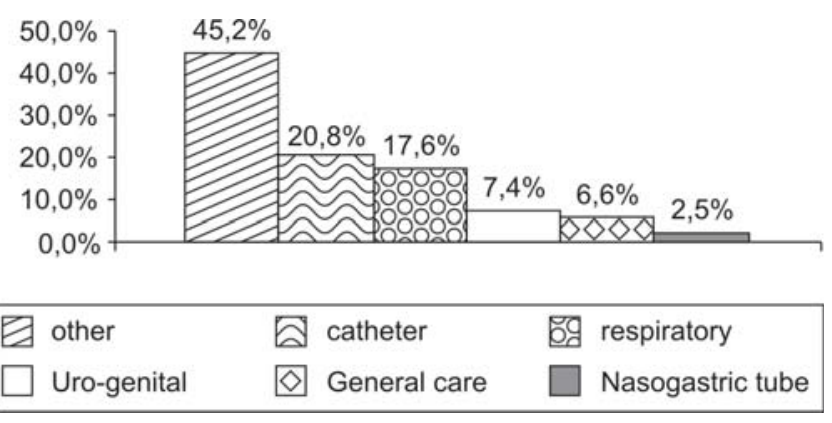

Voss and Widmer have shown that for a complete hand hygiene process and assuming $100 \%$ of compliance, in a model ICU with 14 beds, this would demand 16 hours of total nursing time per day shift, what is impractical for modern ICU's [7].

With this data in mind, along with reports showing that educational measures have only a transient beneficial effect [8,12], alternative strategies to reduce the total time needed for hand hygiene are being attempted. The employment of waterless alcoholic preparations has been shown an effective alternative. Antiseptic properties are similar or even better when compared to conventional agents such as chlorhexidine $[13,14]$, although gel preparations seem to perform worse than liquid formulations according to European standards $[15,16]$. In addition, fewer side effects are reported, enhancing health staff acceptance $[17,18]$. Finally, less time is demanded for hand hygiene, since the need for hand hygiene is minimized. Some elegant studies have shown beneficial effects of the introduction of alcoholic preparations. Studies have shown that the overall hospital wide compliance with hand hygiene [19] as well as specific ICU compliance [20] was enhanced when alcoholic preparations were introduced along with a continuing educational campaign. Pittet et al. [19] showed that alcoholic preparation use increased over time, a phenomenon that was not observed with hand hygiene employing conventional antiseptics. More importantly, $S$. aureus infection/colonization rates and total nosocomial infection rates declined over time [19-21]. An important finding of these studies was that compliance was mostly enhanced in high risk patient care, such as before patient and that the whole campaign, taking into account alcoholic gel consumption, was highly cost-effective care [22].

Bischoff et al. have shown similar results in the United States of America [8]. They were able to show, in both a medical ICU and a cardiac ICU, that hand hygiene compliance failed to rise after an education/feedback program, but increased significantly after introduction of a waterless alcoholic preparation. They showed that one alcohol dispenser per patient even enhanced compliance when compared to a rate 
of 1 dispenser per 4 patients. Finally, a positive effect of the introduction of waterless alcohol based hand hygiene was observed in pediatric ICU's, along with quality improvement interventions [23].

According to the authors, the major limitation in their studies was the fact that randomization was not practical and compliance rates observed after the introduction of alcoholic disinfectants were compared to baseline values. Moreover, the direct role of alcoholic introduction may have been biased by the concomitant educational strategy conducted, although Hugonnet et al. have reported a long lasting effect [21].

To our knowledge, our study is the first to compare different hand hygiene strategies concomitantly. After an educational campaign and a special educational effort to show the beneficial effects of using a waterless alcoholic rub preparation, three units in the same ICU, with health staff submitted to the same educational training, physically apart but with identical design (including number and positioning of sinks) were selected for different hand hygiene strategies. In one of the units, hand hygiene with chlorhexidine, which has been our standard practice for years, was maintained (the compliance rate represents our baseline compliance with hand hygiene). In the second unit, alcohol gel rub bottles (one for each bed) were added to conventional chlorhexidine. In the third unit, only alcohol rub preparations were available in a 1 per bed ratio. Patients were allocated randomly for each unit and no significant different characteristics were observed, including variables that could interfere with health staff activities. In this way, the results were compared in a contemporary way and not with pre-study results. We have shown, in contrast with the above-mentioned studies, very low compliance rates with the alcoholic preparation, little additional effect of adding alcohol disinfection to standard chlorhexidine and a significant difference favoring hand hygiene employing chlorhexidine.

Some aspects must be considered when analyzing such discrepant results. The practice of using water for hand hygiene seemed an important issue for refusing alcohol gel in our study population. In fact, when a questionnaire was used to evaluate the reasons for refusing alcohol gel hygiene, the lack of contact with water was the most frequent reason mentioned by our health staff. This may be an important cultural factor in tropical countries such as Brazil and may be taken into account when attempting to change behavioral practices. On the other hand, compliance with alcoholic preparations has been show to increase significantly over time. Bischoff et al. have documented significant improvement after a six-month observational period [8]. Pittet et al. observed improvement during a 3-year-observational period, although enhanced compliance with hand hygiene using an alcoholic product was evident as early as the first six months of observation, although rates stabilized in the last 18-month period [19]. Hence, the two-month observational period used in our study, even after an intense campaign emphasizing the benefits of the alcoholic preparations may have been insufficient to show an increase in hand hygiene compliance. A longer period of observation is being planned in our ICU to confirm this finding.

Our study has some limitations. First, a longer period of time may be needed to change secular hand hygiene practices and a two-month period could be insufficient. Second, although patient baseline characteristics of the patients were similar when the three units were considered (including admission TISS scores) health staff was not the same and individual practices may have somewhat influenced the results.

The concordant results of studies showing increasing compliance rates with the employment of waterless alcoholic gel preparations foresees a necessary change in hand hygiene practices. Such findings has prompted the development of revised guidelines for hand hygiene in hospital facilities, reserving a central role for the use of alcohol hand rubbing [24]. However, our study shows that the transition from standard hand hygiene practices, employing water and chlorhexidine, may require more time than expected. The abrupt abolition of hand hygiene as part of the hand disinfection process may not be recommended for specific services. Close monitoring of hand disinfection compliance when substituting chlorhexidine for waterless alcoholic rub products is mandatory.

\section{Acknowledgments}

Authors contributions: All the authors have contributed significantly to this work.

\section{References}

1. Guideline for Hand hygiene and Hospital Environmental Control, 1985. Centers for Diseases Control and Prevention.

2. Paulson, SD; Fendler EJ; Dolan, MJ; Williams, RA. A close look at acohol gel as an antimicrobial sanitizing agent. Am J Infect Control 1999;27:332-8.

3. Zaragoza, M; Sallés, M; Gomez, J; Bayas, JM; Trilla, A. Handwashing with soap or alcoholic solutions? A randomized clinical trial of its effectiveness. Am J Infect Control 1999;27:258-61.

4. Goldmann, D; Larson, E. Handwashing and nosocomial infections. N Engl J Med 1992;327:120-22.

5. Graham, M. Frequency and duration of handwashing in an intensive care unit. Am J Infect Control 1990;18:77-81.

6. Pittet, D; Mourouga, P; Perneger, TV. Compliance with handwashing in a teaching hospital. Ann Intern Med 1999;30:126-30.

7. Voss, A; Widmer, AF. No time for handwashing!? Handwashing versus alcoholic rub: can we afford $100 \%$ compliance. Infect Control Hosp Epidemiol 1997;18:205-8.

8. Bischoff, WE; Reynolds, TM; Sessler, CN; Edmond, MB; Wenzel, RP. Handwashing compliance by health care workers. The impact of introducing an accessible, alcohol-based hand antiseptic. Arch Intern Med 2000,160:1017-21.

9. Doebbling BN, Stanley GL, Sheetz CT, et al. Comparative efficacy of alternative handwashing agents in reducing nosocomial infections in intensive care units. N Engl J Med 1992;327:8893.

10. Conly JM, Hill S, Ross J, Lehtzman J, Louie T. Handwashing practices in an intensive unit: the effects of an educational program and its relationship to infection rates. Am J Infect Control 1989;17:330-39. 
11. Austin DJ, Bonten MJM, Weinstein RA, Slaughter S, Anderson $\mathrm{RM}$. Vancomycin-resistant enterococci in intensive care hospital settings transmission dynamics, persistence and the impact of infection control programs. Proc Natl Acad Sci USA 1999;96:6908-13.

12. Simmons B, Brynt J, Neiman K, Spencer L, Arheart K. The role of handwashing in prevention of endemic intensive care unit infections. Infection Control Hosp Epidemiol 1990;11:58994.

13. Rotter ML. Handwashing, hand disinfection and skin disinfection. In: Mayhall G, ed: Hospital epidemiology and infection control, $2^{\text {nd }}$ ed, Baltimore: Williams and Wilkins, 1999:691-709.

14. Girou E, Loyeau S, Legrand P, Oppein F, Brun-Buisson C. Efficacy of handrubbing with alcohol based solution versus standard handwashing with antiseptic soap: randomized clinical trial. BMJ 2002;325:362-66.

15. Kramer A, Rudolph P, Kampf G, Pittet D. Limited efficacy of alcohol-based hand gels. Lancet 2002;359:1489-90.

16. Dharan S, Hugonnet S, Sax H, Pittet D. Comparison of waterless hand antisepsis agents at short application times: raising the flag of concern. Infect Control Hosp Epidemiol 2003;24:16064.

17. Boyce JM, Kelliher S, Vallande N. Skin irritation and dryness associated with two hand-hygiene regimens: soap-and-water hand antisepsis versus hand antisepsis with an alcoholic hand gel. Infect Control Hosp Epidemiol 2000;21:442-48.
18. Graham M, Nixon R, Burrell LJ, et al. Low rates of cutaneous adverse reactions to alcohol-based hand antisepsis solution during prolonged use in a large teaching hospital. Antimicrob Agents Chemother 2005;49:4404-5.

19. Pittet D, Hugonnet S, Harbarth S, et al. Effectiveness of a Hospital Wide programme to improve compliance with hand antisepsis. Lancet 2000;356:1307-12.

20. Gordin FM, Schultz ME, Huber RA, Gill JA. Reduction in nosocomial transmission of drug-resistant bacteria after introduction of an alcoholbased handrub. Infect Control Hosp Epidemiol 2005; 26:650-53.

21. Hugonnet S, Perneger TV, Pittet D. Alcohol-Based Handrub Improves Compliance with Hand antisepsis in Intensive Care Units. Arch Int Med 2002;162:1037-43.

22. Pittet D, Harbarth S, Hugonnet S, Sax, H. Cost-Savings after successful hand antisepsis promotion. $42^{\text {nd }}$ ICAAC, San Diego, 2002, Abstract K-1096.

23. Harbarth S, Pittet D, Grady L, et al. Interventional study to evaluate the impact of an alcohol-based hand gel in improving hand antisepsis compliance. Pediatr Infect Dis J 2002;21:489-95.

24. Boyce JM, Pittet D; Healthcare Infection Control Practices Advisory Committee. Society for Healthcare Epidemiology of America. Association for Professionals in Infection Control. Infectious Diseases Society of America. Hand antisepsis Task Force. Guideline for Hand antisepsis in Health-Care Settings: recommendations of the Healthcare Infection Control Practices Advisory Committee and the HICPAC/SHEA/APIC/IDSA Hand antisepsis Task Force. Infect Control Hosp Epidemiol 2002;23:S3-S40. 